\title{
ELECTRON SELF-ENERGY OF HIGH TEMPERATURE SUPERCONDUCTORS AS REVEALED BY ANGLE-RESOLVED PHOTOEMISSION
}

\author{
M. R. NORMAN ${ }^{\mathrm{a}}, \mathrm{H} \cdot \mathrm{DING}^{\mathrm{a}, \mathrm{b}}$, M. RANDERIA ${ }^{\mathrm{c}}$ and J. C. CAMPUZANO ${ }^{\mathrm{a}, \mathrm{b}}$ \\ ${ }^{a}$ Materials Sciences Division, Argonne National Laboratory, Argonne, IL 60439, USA \\ ${ }^{\mathrm{b}}$ Department of Physics, University of Illinois at Chicago, Chicago, IL 60607, USA \\ ${ }^{\mathrm{c}}$ Tata Institute of Fundamental Research, Mumbai 400005, India
}

\begin{abstract}
In this paper, we review some of the work our group has done in the past few years to obtain the electron self-energy of high temperature superconductors by analysis of angle-resolved photoemission data. We focus on three examples which have revealed: (1) a d-wave superconducting gap, (2) a collective mode in the superconducting state, and (3) pairing correlations in the pseudogap phase. In each case, although a novel result is obtained which captures the essence of the data, the conventional physics used leads to an incomplete picture. This indicates that new physics needs to be developed to obtain a proper understanding of these materials. (C) 1998 Elsevier Science Ltd. All rights reserved
\end{abstract}

Keywords: self energy, photoemission, D. superconductivity

Eleven years after their discovery, the physics of high temperature superconductors is still not well understood because of their complex nature. One of the key tools used to obtain information on these materials has been angle-resolved photoemission spectroscopy (ARPES). Although a surface sensitive probe, ARPES has the advantage of being resolved both in energy and momentum space, thus providing information difficult to obtain from other methods. Given the fact that ARPES measures the single particle spectral function [1], then, in principle, one should be able to obtain the electron self-energy from the data. In some sense, this would 'solve' the high temperature superconductor problem, assuming one had some microscopic theory which produced the same selfenergy.

In this spirit, our group has worked several years now analyzing ARPES data in an attempt to extract useful information about the electron self-energy for high temperature cuprate superconductors. The amount of work done is too extensive to review in this short paper, so we will confine ourselves to three examples. In each case we find a non-trivial result which captures the essence of the data. But in each case, we find that our 'conventional' explanation is in some sense incomplete. We will use this to show that any 'mean field' explanation of the data will always lead to inconsistencies and relate this to the long standing ' $x$ ' versus ' $1+x$ ' debate on the doping dependence of physical quantities. The conclusion is that new physics will need to be developed to obtain a complete picture of the data.

Our first example concerns the determination of the low temperature superconducting gap. Traditionally, workers in ARPES [2] have defined the gap by the midpoint of the leading edge of the spectrum. Although this midpoint is related to the superconducting gap, it is not the same [3]. At low temperatures, and ignoring linewidth broadening and momentum resolution, the midpoint of spectra at the Fermi momentum is the superconducting gap minus the HWHM of the energy resolution, if the gap is large enough so that the Fermi function plays no role. Even with these restrictions, this statement assumes one can equate the photocurrent to the spectral function, and that one knows the Fermi momentum, each of which involves a number of assumptions. We have taken the first step beyond this midpoint criterium in an attempt to give a well-defined meaning to the measurement of the gap by ARPES [4].

We first assert that the measured photocurrent is proportional to the spectral function times the Fermi function, the proportionality constant being the dipole matrix element connecting the initial and final states (the signal above the Fermi energy, due to higher harmonics of the photon beam, is obviously subtracted before making this identification). This assumes (1) the sudden approximation is valid, (2) contributions due to the gradient of the photon vector potential can be ignored, and (3) 'secondaries' (due to inelastic scattering of the photoelectron) are either small or have also been subtracted. Although this seems a lot to stomach at once, there are ways to test this. For instance, if valid, then a frequency integral of the ARPES spectra should be proportional to the momentum distribution function, $n_{\mathbf{k}}$. Our studies [1] indeed indicate that the frequency integrated ARPES data are consistent with such an identification. Exploiting this, a rigorous method can be suggested to determine the Fermi momentum, that point where the gradient of the integrated data (i.e. $\left|\nabla n_{\mathbf{k}}\right|$ ) has a maximum [5]. Doing this, we find a large hole-like Fermi 


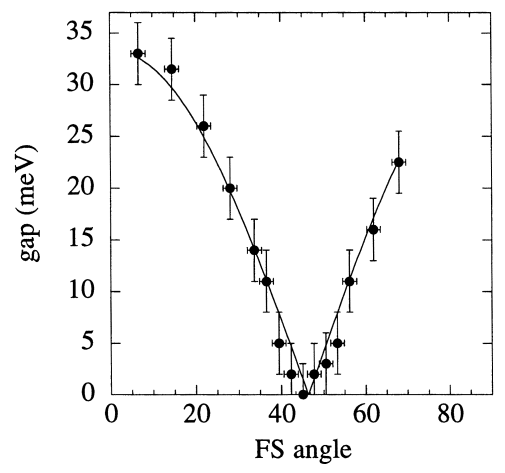

Fig. 1. $Y$ quadrant gap (in meV) versus angle on the Fermi surface (filled circles) compared to a $\mathrm{d}_{x^{2}-y^{2}}$ gap (—).

surface centered about the $(\pi, \pi)$ points of the square lattice Brillouin zone with a volume consistent with $1+x$ [6].

We next need some model spectral function by which to fit the data. Since we wish to determine the BCS gap, then it is natural to use a BCS spectral function. At low temperatures we are fortunate, in that the leading edge of the spectrum is resolution limited. This implies that the imaginary part of the electron self-energy is small at frequencies of order the superconducting gap, and thus there is some justification for using a BCS-like ansatz (limitations of this picture will be discussed in our next example). For frequencies beyond the gap, however, selfenergy effects cannot be ignored. One sees this in the data as a large non-quasiparticle peak contribution to the spectrum. Whether all of this weight is part of the true spectral function or not (i.e. secondaries) is still a matter of debate. In our case, we simply subtracted this incoherent part by assuming it could be treated as 'secondaries' (using the standard Shirley procedure). In practice, for extracting the gap, this makes little difference since one is fitting only the leading edge of the spectrum, which is not much affected by this subtraction (though this subtraction becomes more of an issue as the temperature is raised). But this does illustrate the point made at the beginning. Although by doing this subtraction we are able to fit the data to a BCS spectral function, and thus obtain a reliable estimate of the BCS gap, we have in essence only obtained a single number. Although it is a very useful number, as we will see, it encapsulates only one aspect of the very interesting ARPES spectra in the superconducting state.

Our first attempt at this procedure revealed a gap in Bi2212 which was a strong function of the Fermi momentum [4], in support of earlier work by the group of Shen [2]. Unlike this earlier work, evidence for two zeros of the gap as a function of momentum (per zone quadrant), rather than the single zero expected for a d-wave order parameter, was found. At that time, we suggested two possibilities for interpreting this: (1) an anisotropic s-wave gap, or (2) a d-wave gap which was

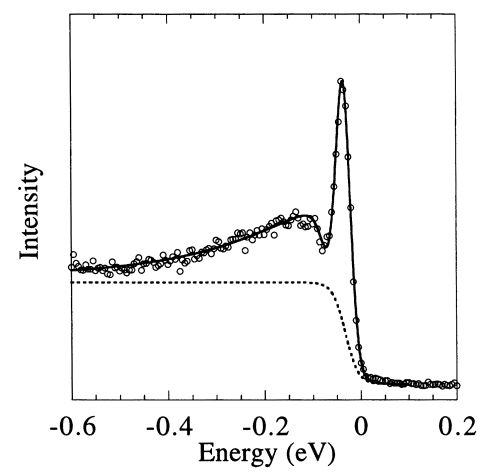

Fig. 2. Comparison of SC data at $(\pi, 0)$ to a model fit based on electrons interacting with a collective mode.---, an assumed background contribution.

either being measured on the true Fermi surface or one of the ghost images of the Fermi surface (the ghosts due to diffraction of the outgoing photoelectrons by the incommensurate $\mathrm{BiO}$ superlattice) depending on what particular value of momentum one was measuring. Subsequently, by exploiting the photon polarization dependence of the dipole matrix elements, we were able to show that explanation (2) was actually the correct one $[7,8]$. As extensively discussed in these papers, the superlattice complications can be avoided by measuring the gap in the $Y$ quadrant of the Brillouin zone. Doing so reveals a gap which beautifully follows the behavior predicted for an order parameter with $d_{x^{2}-y^{2}}$ symmetry [8] (see Fig. 1). In fact, one learns more than this. Since the data follow the form $\cos \left(k_{x} a\right)-\cos \left(k_{y} a\right)$ quite closely, this indicates that the pairing interaction is fairly local in real space. Data taken on another optimally doped sample where the large gap region was sampled more closely [8] actually indicate the presence of a weak maximum in the gap at locations on the Fermi surface connected by $(\pi, \pi)$ wavevectors. Similar effects have been seen in calculations where spin fluctuations are considered as the pairing mechanism.

As said above, even though a lot of useful information is obtained from knowing the value of $\Delta_{\mathbf{k}}$, it is only a small part of the overall story. This can be seen in Fig. 2, where the spectrum at the $(\pi, 0)$ point of the zone for a slightly overdoped sample at low temperatures is shown. Rather interestingly, this spectrum agrees with that of the normal state for energies beyond about $90 \mathrm{meV}$, which is equivalent to stating that the self-energies agree beyond this energy. For lower energies, though, one sees a dramatic departure of the superconducting state spectra from the normal state one, as first noted by Dessau et al. [9]. The superconducting (SC) state spectrum first drops (thus leading to a dip/hump structure) then rises to form a sharp, essentially resolution limited, quasiparticle peak. Since this change in behavior is all occuring on the scale of the energy resolution, this indicates that the imaginary part of the self-energy $(\operatorname{Im} \Sigma)$ must drop from its large 
normal state value to a small value over a narrow energy range. Fits we have done using model self-energies reveal that the drop in $\operatorname{Im} \Sigma$ must be rather abrupt, essentially a step edge (the standard d-wave prediction of crossing over from $\omega$ to $\omega^{3}$ is too weak to give a dip). In fact, the observed dip is so deep, it is best fit by a peak in $\operatorname{Im} \Sigma$ followed by a rapid drop.

There are a number of consequences of such behavior. By Kramers-Kronig transformation, a step in $\operatorname{Im} \Sigma$ implies a peak in $\operatorname{Re} \Sigma$. Such a peak will lead to an additional mass renormalization relative to the normal state which acts to suppress the quasiparticle dispersion. This explains the rather puzzling observation that the quasiparticle peak does not appear to disperse much when moving away from the $(\pi, 0)$ point. In fact, data on a number of our samples indicate that a sharp low energy peak is still present when moving towards the $(0,0)$ point even when the higher binding energy feature (the hump) has begun to disperse (the hump dispersion rapidly approaches the dispersion of the single broad peak seen in the normal state). This result was so puzzling that the data were not published for several years. The peak in $\operatorname{Re} \Sigma$, however, naturally explains this, since it can lead to a low energy quasiparticle pole even when the normal state binding energy moves well away from the Fermi energy. We note that a step edge in $\operatorname{Im} \Sigma$ is equivalent to the problem of an electron interacting with a dispersionless mode, as previously treated by Engelsberg and Schrieffer [10]. Such an interaction leads to the prediction of a spectral function composed of two features (peak and hump) whose dispersion is remarkably similar to that extracted from the data [11]. The crucial difference here is that this behavior appears only in the superconducting state, and thus the mode is not a phonon. Rather, it must be of collective origin. Detailed calculations we have done of a superconducting electron interacting with a dispersionless mode give a good description of the data [12], with the peak in $\operatorname{Im} \Sigma$ due to the peak in the SC density of states. They also give a good fit to the observed dispersion of the two features.

Microscopically, these findings imply that the dominant contribution to the electron self-energy is from electron-electron scattering processes. The low frequency reduction in $\operatorname{Im} \Sigma$ is a consequence of the gapping of the spectrum causing the scattering (i.e. $\alpha^{2} F$ ) by the superconducting gap (the $2 \Delta$ effect first discussed by Kuroda and Varma [13]). To get a step edge, though, one must assume that the gapped weight shows up as a sharp mode inside of this ' $2 \Delta$ ' gap. Recent spin fluctuation theories have indeed predicted such behavior [14]. Fits to the data indicate a mode with an energy of $41 \mathrm{meV}$, equivalent to that of the collective mode seen by neutron scattering in $\mathrm{YBCO}$ [15]. Whether this is a coincidence or not remains to be seen. The YBCO neutron scattering data indicate that the mode is associated with $(\pi, \pi)$ scattering events, whereas in the ARPES data the step edge in $\operatorname{Im} \Sigma$ implies dipsersionless behavior. On the other hand, the low energy ARPES peak exists over about the same momentum range along $(\pi, 0)-(\pi, \pi)$ as it does along $(\pi, 0)-(0,0)$. As these directions are related by a $(\pi, \pi)$ translation, the ARPES data also indicate that $(\pi, \pi)$ scattering is indeed playing an important role. This is most obviously seen in the fact that the dip/hump structure is most pronounced in spectra at the $(\pi, 0)$ points, as recently emphasized by Shen and Schrieffer [16].

It is important to remark that the above description is incomplete. In Fig. 2, we show that our model gives a very good fit to the spectra, but at a price. The price is that a large 'background' contribution has to be subtracted off the data. This background is modeled by a step, and is essentially equivalent to the total ARPES spectra for unoccupied states (with the step edge at the Fermi energy in the normal state, but pushed back by $\Delta$ in the superconducting state). In reality, there are indications that most if not all of this background is part of the true spectral function. This has led to a recent speculation that this large background actually represents the instability of the photohole to decay into spinons and holons [17]. In such a model, the quasiparticle peak represents a bound state split from this continuum. To look into these matters in more detail, we have recently attempted to extract the actual experimental self-energy by direct inversion of the ARPES data. This inversion reveals the predicted peak and step edge in $\operatorname{Im} \Sigma$ of our model, as well as the peak in $\operatorname{Re} \Sigma[18]$.

We now ask ourselves the question of how the above picture changes as the doping is reduced, moving towards the Mott insulating phase. What is found is that the low temperature gap again has the expected d-wave form [19]. On the other hand, the spectrum does change significantly as the doping is reduced. The quasiparticle peak becomes smaller and the hump becomes more pronounced, moving to higher binding energy. Perhaps the most significant change is seen upon heating the sample. In overdoped Bi2212, the superconducting gap is observed to close at or near $T_{\mathrm{c}}$. Unpublished fits we have done similar to those of Fig. 1 (which are obviously more suspect as the temperature is raised) reveal a gap whose $T$ dependence is much flatter than the BCS prediction, which then closes rapidly near $T_{\mathrm{c}}$. This is accompanied by a strong increase in the low frequency broadening back to its large normal state value (the broadening is found to drop approximately like $T^{6}$ below $T_{\mathrm{c}}$, similar to what is seen in conductivity measurements, and again a strong indication of the electron-electron scattering origin of $\Sigma$ ). In the underdoped case, however, something quite different occurs. The spectral gap is seen to exist well above $T_{\mathrm{c}}$, only disappearing at a higher temperature (denoted $T^{*}$ ) [19-21] and has a similar anisotropy as that seen below 
$T_{\mathrm{c}}$. The gap as measured by the midpoint of the leading edge goes smoothly through $T_{\mathrm{c}}$ [19] indicating that the gap above $T_{\mathrm{c}}$ has the same origin as the gap below $T_{\mathrm{c}}$, as predicted by theories with pairing correlations above $T_{\mathrm{c}}$ [22].

This so-called pseudogap has been seen in a variety of other measurements, most of them predating the ARPES ones. Its origin is a matter of intense debate and encapsulates one of the most fundamental issues of high temperature superconductivity: how the unusual superconducting state seen in the cuprates evolves into the equally unusual Mott insulating state. Again, the advantage of ARPES is that it provides both momentum and frequency resolved information. What does it find? First, the quasiparticle peaks appear only below $T_{\mathrm{c}}$ (i.e. not below $T^{*}$ ), and again one finds that the low frequency broadening drops off roughly as $T^{6}$ below $T_{\mathrm{c}}$. Above $T_{\mathrm{c}}$, the spectra near $(\pi, 0)$ are quite unusual, being rather flat, but with a sharp leading edge with a large gap (note, this leading edge gap discussed in Refs. [19, 21] is not the same as the hump position discussed in Ref. [20], a point of confusion in the literature). Second, this leading edge gap, as characterized by its midpoint, smoothly evolves through $T_{\mathrm{c}}[19,23]$ implying that it is of the same origin as the superconducting gap. Third, the low temperature gap actually increases as the doping decreases, reflecting the increase of $T^{*}$ with underdoping [23-25], again showing the strong connection between the superconducting gap and pseudogap. Fourth, the pseudogap has a similar anisotropy above $T_{\mathrm{c}}$ to that below $[19,21]$. Fifth, the pseudogap is tied to the normal state Fermi surface [26], as expected if the gap were of pairing origin, rather than of CDW or SDW origin.

Recently, we have looked again into the anisotropy issue by taking temperature sweeps at different $\mathbf{k}$ points on the Fermi surface. We found something quite unusual, in that the pseudogap disappears at different temperatures for different $\mathbf{k}$ [23]. This means that the d-wave node below $T_{\mathrm{c}}$ becomes a gapless arc above $T_{\mathrm{c}}$ which expands in length with temperature, until the entire Fermi surface is recovered at $T^{*}$. The evolution is smooth, as predicted by theories with d-wave pairing correlations [27], rather than the abrupt change one would expect if the gap above $T_{\mathrm{c}}$ were of different origin than the one below $T_{\mathrm{c}}$.

Closer inspection, though, reveals that more is going on. This is most clearly seen by employing a recent method we have suggested for removing the effects of the Fermi function from ARPES data [23]. If one assumes the spectral function is particle-hole symmetric, a mild assumption for spectra at $k_{\mathrm{F}}$ over a sufficiently narrow energy range about the Fermi energy, then one can formally eliminate the Fermi function from the data by summing the ARPES intensity at positive and negative energies (with respect to the chemical potential). By doing so, one acquires a dramatic visual picture of what

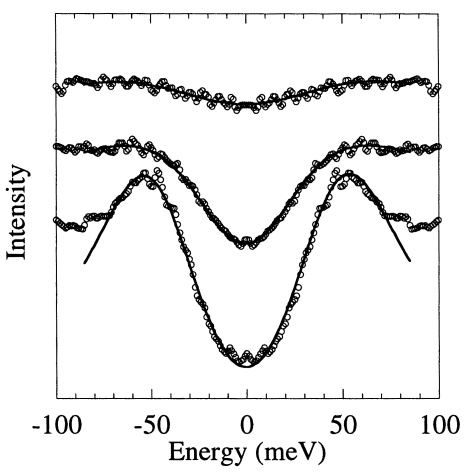

Fig. 3. Symmetrized data on a $T_{\mathrm{c}}=83 \mathrm{~K}$ sample at the $(\pi, 0)-$ $(\pi, \pi)$ Fermi crossing at three temperatures $(14 \mathrm{~K}, 90 \mathrm{~K}$, and $170 \mathrm{~K}$ ) compared to model fits based on pairing fluctuations.

is going on. Such symmetrized data are shown in Fig. 3 at the $(\pi, 0)-(\pi, \pi)$ Fermi crossing for a moderately underdoped $\left(T_{\mathrm{c}}=83 \mathrm{~K}\right)$ sample. One clearly sees from this that the spectral gap fills in rather than closes. In contrast, halfway along the Fermi surface between $(\pi, 0)$ to $(\pi, \pi)$ the behavior is quite different, as the gap is seen to actually close (at a temperature between $T_{\mathrm{c}}$ and $T^{*}$ ) rather than fill in. The momentum dependence of the electron self-energy is highly non-trivial.

These results have motivated us to find a model selfenergy which captures the unusual behavior seen near the $(\pi, 0)$ point in the pseudogap phase. One which reproduces the low energy data quite well (see Fig. 3) is of the form $-i \Gamma_{1}+\Delta^{2} /\left(\omega+i \Gamma_{0}\right)$ [28]. BCS theory is recovered by setting $\Gamma_{0}$ to zero. The surprising finding is that $\Gamma_{0}$ is proportional to $T-T_{\mathrm{c}}$ (and thus zero below $T_{\mathrm{c}}$ ), with $\Delta$ essentially $T$ independent (the latter having been inferred earlier from specific heat data in YBCO [29]). $T^{*}$ then corresponds to where $\Delta=\Gamma_{0}(T)$, i.e. although the spectral gap closes, $\Delta$ is still non-zero. The interesting point is that there is only one known quantity which is proportional to $T-T_{\mathrm{c}}$, the inverse Cooper pair lifetime [30]. This almost certainly means that the pseudogap is due to pairing correlations [28]. In CDW or SDW type theories, the quantity $T-T_{\mathrm{c}}$ would not naturally arise. In fact, the above form for the self-energy can be motivated by a $t$-matrix calculation of the self-energy due to pairing fluctuations [28]. The derivation is especially transparent in the limit where the bands are dispersionless, which thus motivates why the behavior is seen only near the $(\pi, 0)$ points of the zone.

This 'zero dimensional' behavior again emphasizes the unconventional nature of the cuprates. A model having these characteristics has been recently proposed by Geshkenbein et al. [31], who emphasized that such behavior can explain the existence of large pairing correlations without the large expected signatures of fluctuational conductivity or diamagnetism. In essence, the states near $(\pi, 0)$ have no measurable Fermi velocity and not even a remnant of a quasiparticle peak in the 
pseudogap phase, and consequently they do not contribute to the supercurrent response. Any type of mean field treatment would therefore be totally inadequate. Although these electrons near $(\pi, 0)$ have a large $\Delta$, they have no $\Psi$ in the Ginzburg-Landau sense. As the doping decreases, the anomalous region expands, eventually taking over the entire zone, giving rise to the non-superconducting, insulating state (data taken on low $T_{\mathrm{c}} \mathrm{Bi} 2212$ samples show this type of behavior $[19,26])$.

Any mean field description of the above would force one again into the two gap picture, which is inconsistent with the data, in that the gap for each $\mathbf{k}$ smoothly evolves through $T_{\mathrm{c}}$, and at low temperatures has an anisotropy completely consistent with a simple d-wave order parameter. This same kind of inconsistency is also seen if one attempts to force a Fermi liquid picture in the underdoped superconducting state [32], and is related to the ' $x$ ' versus ' $1+x$ ' debate which has been prevalent in the cuprate literature over the past decade, a debate which encapsulates the unconventional nature of the cuprates. Is it $x$, or $1+x$ ? In some sense, the ARPES data say it is both, analogous to quantum mechanics whose objects behave like waves or particles depending on what question one is asking. Is there a large Fermi surface enclosing a volume $1+x$, even for reduced doping? The answer is yes, but [26]. The 'but' is due to the fact that one can quite reasonably define a Fermi crossing along $(\pi, 0)$ $(\pi, \pi)$ due to the intensity drop in the spectra (the $\left|\nabla n_{\mathbf{k}}\right|$ maximal argument discussed earlier), but this hides the fact that there is no true dispersion in the conventional band theory sense. In essence, states near the $(\pi, 0)$ points do not behave like a normal Fermi liquid, and the rest form a liquid whose effective volume is increasingly reduced as the doping is reduced, leading to $x$-like scaling (note, this is not the same as the 'mean field' $x$ picture with small hole pockets, something we find no evidence for). This behavior is perhaps most exhaustively discussed in the recent book of Anderson [33].

In conclusion, much useful information concerning the electron self-energy can be extracted from an analysis of the ARPES data. In each example studied above, a conventional, but exotic, explanation is found (d-wave superconducting gap, collective mode in the superconducting state, pairing correlations above $T_{\mathrm{c}}$ ). But, in each case, the conventional explanation, although capturing the essence of the data, is incomplete. This indicates that new physics needs to be developed before a true understanding of the cuprates is achieved.

Acknowledgements-This work was supported by the US Department of Energy, Basic Energy Sciences, under contract W-31-109-ENG-38, the National Science Foundation DMR 9624048, and DMR 91-20000 through the Science and Technology Center for Superconductivity.

\section{REFERENCES}

1. Randeria, M., et al., Phys. Rev. Lett., 1995, 74, 4951.

2. Shen, Z.-X., et al., Phys. Rev. Lett., 1993, 70, 1553.

3. Fehrenbacher, R., Phys. Rev., 1996, B 54, 6632.

4. Ding, H., et al., Phys. Rev. Lett., 1995, 74, 2784.

5. Campuzano, J.C., et al., Phys. Rev., 1996, B53, R14737.

6. Ding, H., et al., Phys. Rev. Lett., 1996, 76, 1533.

7. Norman, M.R., et al., Phys. Rev., 1995, B52, 15107.

8. Ding, H., et al. Phys. Rev., 1996, B54, R9678.

9. Dessau, D.S., et al., Phys. Rev. Lett., 1991, 66, 2160.

10. Engelsberg, S. and Schrieffer, J.R., Phys. Rev., 1963, 131, 993.

11. Norman, M.R., et al., Phys. Rev. Lett., 1997, 79, 3506.

12. Norman, M.R., Ding, H., Phys. Rev. B, 1998, 57, R11089.

13. Kuroda, Y. and Varma, C.M., Phys. Rev., 1990, B 42, 8619.

14. Pao, C.-H. and Bickers, N.E., Phys. Rev., 1995, B 51, 16310.

15. Rossat-Mignod, J., et al., Physica C, 1991, 185-189, 86.

16. Shen, Z.-X. and Schrieffer, J.R., Phys. Rev. Lett., 1997, 78, 1771 .

17. Laughlin, R.B., Phys. Rev. Lett., 1997, 79, 1726.

18. Norman, M. R. et al., cond-mat/9806262.

19. Ding, H., et al., Nature, 1996, 382, 51.

20. Marshall, D.S., et al., Phys. Rev. Lett., 1996, 76, 4841.

21. Loeser, A.G., et al., Science, 1996, 273, 325.

22. Randeria, M., Trivedi, N., Moreo, A. and Scalettar, R.T., Phys. Rev. Lett., 1992, 69, 2001.

23. Norman, M.R., et al., Nature, 1998, 392, 157.

24. Harris, J.M., et al., Phys. Rev., 1996, B54, R15665.

25. Miyakawa, N., et al., Phys. Rev. Lett., 1998, 80, 157.

26. Ding, H., et al., Phys. Rev. Lett., 1997, 78, 2628.

27. Engelbrecht, J.R., Nazarenko, A., Randeria, M., Dagotto, E., Phys. Rev. B, 1998, 57, 13406.

28. Norman, M.R., Randeria, M., Ding, H., Campuzano, J.C., Phys. Rev. B, 1998, 57, R11093.

29. Loram, J.W., et al., J. Supercond., 1994, 7, 243

30. Abrahams, E. and Tsuneto, T., Phys. Rev., 1966, 152, 416.

31. Geshkenbein, V.B., Ioffe, L.B. and Larkin, A.I., Phys. Rev., 1997, B 55, 3173.

32. Lee, P.A. and Wen, X.G., Phys. Rev. Lett., 1997, 78, 4111

33. P.W. Anderson, The Theory of Superconductivity in the High $T_{\mathrm{c}}$ Cuprates, Princeton University Press, Princeton, 1997. 